九州大学学術情報リポジトリ

Kyushu University Institutional Repository

Staat, Nation und Familie : Zum Verhältnis von Feminismus und Nationalstaat in Japan, 19181945

Germer, Andrea

Faculty of Social and Cultural Studies, Kyushu University

http://hdl. hand le. net/2324/22034

出版情報: Familienangelegenheiten, pp.21-47，2007. German Institute for Japanese Studies バージョン：

権利関係 : 


\title{
STAAT, NATION UND FAMILIE: ZUM VERHÄLTNIS VON FEMINISMUS UND NATIONALSTAAT IN JAPAN, 1918-1945
}

\author{
Andrea Germer
}

Abstract: Historical research on nationalism in Japan has shown how different concepts of an integrative political state and a genealogical ethnic nation competed since the Meiji period. Initially, concepts of cultural and ethnic nationalism served for populist attacks against the political state in modern Japan; by the 1920s, however, they were also utilized by the state to serve functions of identification with and justification of imperial Japan. In this paper, I examine women's discourses on motherhood and family and analyze how some feminists since the Taishō period have directed their demands towards the Japanese political state (as a welfare state) and how others expounded anti-statist views and claimed the ethno-cultural nation as the backup for their feminist agenda. In 1930s and 1940s Japan, the convolution of ethnonational rhetoric with welfare state's gendered politics and the partial integration of women into political offices were two important strategic elements that explain the subsequent cooptation of different groups of feminists by the wartime government.

\section{EINFÜHRUNG: NATION UND GESCHLECHT}

Die feministische Theorie und Geschichtsforschung beschäftigt sich im Hinblick auf die Nation und vor allem auf Nationalismen nicht mehr nur mit Fragen der Ausschließung von Frauen, sondern ebenso mit dem Phänomen ihrer widersprüchlichen Integration in moderne soziale, politische und kulturelle Systeme. ${ }^{1}$ In Japan manifestiert sich diese

* Für konstruktive Kommentare zur Überarbeitung dieses Artikels möchte ich dem Herausgeber und den anonymen Gutachtern recht herzlich danken; ebenso wie den Veranstaltern des III. Symposiums Asiatische Selbstbehauptungsdiskurse an der Universität Erlangen (in Zusammenarbeit mit der Universität Osaka und dem DIJ Tokyo), auf deren Einladung hin ich 2002 die erste Fassung dieses Textes zur Diskussion stellen konnte.

1 Siehe unter anderen Yuval-Davis (1997), Anthias und Yuval-Davis (1992), Yuval-Davis und Anthias (1989). Zur japanischen Geschichtsdiskussion siehe Igeta (2000) und Ueno (2004). In Deutschland hat vor allem die Diskussion um die Rolle der Frauen im Nationalsozialismus die Sicht auf Frauen verändert. Von Opfern wurden sie zu Täterinnen bzw. Mittäterinnen in einer oftmals sehr moralisierend geführten Debatte um die "Schuldfrage" deutscher Frauen (Koonz 1987, 1992, Bock 1989, 1992, 1993, Böltken 1995). 
erweiterte Perspektive in einer Frage, die in der Forschung heftig diskutiert wird und auch für das Verständnis der japanischen Geschichte wichtig ist: der Integration verschiedener Strömungen der Frauenbewegung in die nationalstaatliche Mobilisierung zum Asiatisch-Pazifischen Krieg.

Dieser Frage geht auch der vorliegende Aufsatz nach, indem er Diskurse der japanischen Frauenbewegung aufarbeitet, in denen Geschlechtsidentität, Mutterschaft, Familie und Nationalstaat unterschiedlich miteinander verknüpft und konzeptionalisiert werden. Hier wird die Grundthese entwickelt, dass verschiedene Flügel der Frauenbewegung sich in diesen Debatten der Vorkriegszeit auf unterschiedliche Instanzen des Staates als institutionellem Gebilde auf der einen Seite und des ethnischen Nationalismus auf der anderen beriefen. Beiden Flügeln wurde von nationalstaatlicher Seite entgegengekommen, als die japanische Regierung sich in den 1930er und 1940er Jahren zur Mobilisierung des Volkes und zur Legitimierung seiner militaristischen Expansionspolitik einerseits einer ethno-nationalistischen Rhetorik bediente, die Mutterschaft und Familie im Dienste der Nation ideologisch aufwertete, und andererseits Feministinnen die Option eröffnete, in politischen Gremien der staatlichen Generalmobilisierung mitzuarbeiten.

Über den Zusammenhang von Nationsbildung und Geschlecht sind in den letzten Jahren eine Reihe wichtiger Studien erschienen. Für Europa wurde herausgearbeitet, dass die bürgerliche Gesellschaft im 18. Jahrhundert, die Entstehung der Nation und die radikale Unterscheidung der Geschlechter sich als politische Phänomene zeitgleich herausgebildet haben und in ihrer theoretischen Konzeptualisierung fundamentale Übereinstimmungen aufweisen (Blättler 2000: 109, Connell 2000: 3-4, Mosse 1997: 27, Hausen 1992). Die Ausbildung einer radikal differenten Geschlechtsidentität (Blättler 2000: 110) und die Durchsetzung des nationalen Bewusstseins (Gellner 1983) werden als Konsequenz der Umstellung von einer schichtspezifisch definierten auf eine funktionell ausdifferenzierte Gesellschaft verstanden. Variationen dieser modernisierungstheoretischen These wurden im Falle Japans für die Meiji-zeitliche Abschaffung des Ständesystems und die einheitliche Einführung des patriarchalen Familiensystems (ie seido) sowie weiterer dezidiert geschlechtsspezifischer politischer und gesellschaftlicher Verordnungen jener Zeit vertreten (Getreuer-Kargl 1997, Takamure 1954-58; vgl. auch Sievers 1983: Kap. 2). Mit dem internationalen Druck, dem sich Japan durch die sogenannten Ungleichen Verträge ausgesetzt sah, und mit der Aufhebung der Standesgrenzen wurden Nation und Geschlecht auch in Japan zu wesentlichen Quellen der 
Selbstidentifikation und zu zwei miteinander verschränkten zentralen „Leitdifferenzen“. ${ }^{2}$

Blättler (2002: 2) analysiert das Ineinandergreifen des Nationen- und des Geschlechterdiskurses im Europa des späten 18. und frühen 19. Jahrhunderts und konstatiert, dass „mit Anbruch der politischen Moderne nationale Identität vermittelt durch Geschlechtsidentität und Geschlechtsidentität vermittelt durch nationale Identität in die persönliche Selbstauslegung eingegangen sind". Mit der Einbeziehung kapitalistischer Entwicklungen, vor allem der Schaffung und Existenz von Kolonien, wurde sowohl für die westliche Welt (Enloe 1989, Mamozai 1990, Mies 1990) als auch für Japan (de Bary 1997: 3) auf die globalen Dimensionen der sich herausbildenden nationalen Geschlechterordnungen und ihren Zusammenhang mit dem expansiven Nationalstaat hingewiesen. Die globalen Rück- und Wechselwirkungen dieser geschlechtsspezifischen und nationalen Prozesse hat Conrad (2002) thematisiert und mit der Forderung nach einer transnationalen Geschichtsschreibung verbunden.

Die jeweilige Form der Integration von Frauen in den Prozess der Nationsbildung ist von Land zu Land verschieden. Die Grundlage der Integration im Meiji-zeitlichen Japan bestand darin, dass die Regierenden im Unterschied zur vorausgegangenen Tokugawa-Zeit (1600-1867) überhaupt erstmals auf die aktive geistige Beteiligung des Volkes an der Realisierung des nationalen Projektes abzielten (Fujitani 1993: 99). In den japanischen Moralerziehungslehrbüchern der späten Meiji-Zeit wurde mit dem Konzept der "guten Ehefrau und weisen Mutter" (ryōsai kenbo) das Schicksal des Nationalstaates mit der als traditionell und natürlich imaginierten Rolle der Frau als Mutter und mit ihrem Platz in der Familie verbunden (Nagahara 1985: 194, Koyama 1991). Das Konzept selbst ist ein Konglomerat aus westlichen Idealen von "domesticity" und japanisch-konfuzianistischen Elementen, welche die Rolle der Frauen in der Familie als sparsam waltende Hausfrau mit dem auch ökonomischen Dienst für den Staat verknüpfen.

Dass dieses Rollenideal nicht zwingend zum Nationsbildungsprozess gehört, zeigen andere Beispiele. So propagierte der türkische Nationalismus, der Anfang des 20. Jahrhunderts zur Kemalistischen Republik führte, in Distanzierung vom Islam die Frauenbefreiung als Parameter des kulturellen Nationalismus. Hier war es umgekehrt gerade die weibliche Emanzipation, die als konstitutiv für die Bildung der Nation verstanden wurde (Kandiyoti 1989, Kandiyoti 1991: 43). In späteren, antikolonialistischen nationalen Befreiungskämpfen des 20. Jahrhunderts wurde der aktive und kämpferische Beitrag von Frauen propagiert und die nationale

2 Dieser Begriff stammt von Blättler (2002), die Nation und Geschlecht als zentrale Leitdifferenzen in modernen Gesellschaften bewertet. 
Befreiung oftmals in Darstellungen von Frauen mit dem Gewehr in der Hand und einem Kind auf dem Rücken inszeniert (Mies 1990: 225-26). McClintock hat sowohl für antikoloniale nationalistische Ideen als auch für ihre Umsetzung in Form von post-kolonialen Nationalstaaten darauf hingewiesen, dass die Rhetorik nationaler Einheit immer schon mit der Idee und Politik der geschlechtlichen Differenz befrachtet ist. Sie kommt zu dem Schluss, dass feministische Agenden in nationalen Befreiungskämpfen zwar aufgenommen werden, dabei jedoch primär zum Zwecke der Mobilisierung von Frauen dienen: „Nowhere has feminism in its own right been allowed to be more than the maidservant to nationalism" (McClintock 1997: 110). Andererseits haben Feministinnen, wie im Folgenden zu zeigen ist, nationalistische Rhetorik und Ideen auch für die Verfolgung ihrer eigenen politischen Ziele genutzt.

Um zu verstehen, wie unterschiedliche geschlechtsspezifische Formen der Integration des Volkes in das nationale Projekt entstanden, auf welche Weise Geschlecht sich als Leitdifferenz entwickelte und wie die geschlechtlich identifizierten Individuen ihrerseits auf Abgrenzungs- und Einschließungsangebote reagierten bzw. selbst an diesen Identifikationsprojekten mitwirkten, ist es angebracht, die Unterscheidung von Staat und Nation zu thematisieren.

\section{STAAT UND NATION}

Die neuere auch auf Japan bezogene Forschung hat herausgearbeitet, dass in modernen Nationalismen oft unterschiedliche Konzepte eines integrativen politischen Staats und einer genealogischen ethnischen Nation miteinander konkurrierten. ${ }^{3}$ Solange diese konzeptionelle Unterscheidung nicht erfasst wurde, war Nationalismus ein Konzept, das gezwungenermaßen auf dem Boden des Vergleichs mit dem Westen beruhte (de Bary 1997: 6), von dem aus die unterschiedlichsten Denker und Denkerinnen in der japanischen Geistesgeschichte ihre Theorien entwarfen. Die gegen das Ausland und auf das nationale Wohl ausgerichtete Befreiung war Gedankengut so unterschiedlicher Intellektueller und politischer Aktivisten wie des Aufklärers Fukuzawa Yukichi, des Anarchisten Kōtoku Shūsui (Harootunian 1979), der feministischen Sozialistin Fukuda Hideko (Hane 1988), des Nationalgelehrten Motoori Norinaga (Matsumoto 1970) oder

3 Doak $(1996,1997)$ und Toby (2001). Letzterer nimmt die Unterscheidung von Staat und Nation in Bezug auf die politische Organisation der Tokugawa-Zeit vor und bezeichnet diese als "protonation" (Toby 2001: 227, 230). Siehe auch Karlin (2002: 43). 
der Frauengruppe für Moralreformen Fujin Kyōfūkai (Suzuki 1992), weshalb für Harootunian (1979: 57) die Bezeichnung "nationalistisch“ als Unterscheidungsbegriff nicht brauchbar schien: „[...] the concept of nationalism has failed to yield an operational method adequate to organizing and examining specific experiences".

Hingegen leistet eine begrifflich-analytische Unterscheidung von Nation und Staat (Smith 2001, Chatterjee 1993, Radhakrishnan 1992) sowie eine historisch-empirische Unterscheidung zwischen politischem und ethnischem Nationalismus, wie Doak (1996: 97) sie für Japan herausgearbeitet hat, eine wertvolle Differenzierung. Der Staat bezieht sich als Apparat auf die Sphäre der Regierung und Verwaltung, wohingegen die Nation eine kulturelle und im Falle Japans ethnisch begründete Einheit imaginiert, die auf eine gemeinsame mythische Abstammung und Geschichte, ein Schriftsystem sowie Riten, Familien- und Verwandtschaftssysteme rekurriert. In ihrer Analyse europäischer philosophischer Texte zum Nationalstaat (Hegel, Fichte, Rousseau) hat Blättler (2000: 113) herausgearbeitet, dass die Doppelstruktur von Staat und Nation die "komplementaritätstheoretisch formulierte Geschlechterdualität ziemlich genau" abbildet, indem der Staat sich mit den männlich konnotierten Prinzipien wie Rationalität, Wille, Gesetz, Krieg und dem Ausdruck Vaterland verbindet, während die Nation stärker mit den weiblich konnotierten Elementen wie (kulturelle und ethnische) Materialität, Passivität, Sprache und dem Ausdruck Mutterland assoziiert wird (vgl. auch Chatterjee 1993). Diese Ansätze und Unterscheidungen sollen im Folgenden kritisch auf Diskurse der japanischen Frauenbewegung bezogen werden. Dabei soll überprüft werden, wie einzelne Frauen und Frauengruppen auf Identifikationsangebote des Nationalstaates reagierten, sie mitgestalteten oder sie ablehnten.

"Where, if not Japan, are the nation and the state more identical?", fragt Doak (1997: 283) und beschreibt den Prozess, in dem der Staat in der MeijiZeit zunächst Ziel populistischer Attacken des kulturellen oder ethnischen Nationalismus war, sich dann allmählich der ethno-nationalistischen Rhetorik bediente und die Nation und der Staat schließlich zu einem identischen und identifikatorischen Komplex zusammenschmolzen. Diesen diskursiven Prozess der Rechtfertigung staatlicher Strategien mittels ethnonationalistischer Rhetorik nennt Thomas (1998: 114) eine seit Beginn des 20. Jahrhunderts verfolgte Strategie der "Naturalisierung des Nationalen" (naturalizing nationhood). Beispielhaft führt sie aus, wie als natürlich verstandene religiöse Bindungen mit der radikalen Reduzierung der Anzahl von Schreinen auf lokaler Ebene und ihrer Zusammenlegung aufgelöst und in Ausrichtung auf den tennō und die Zentralisierung auf den Staats-Shintō hin wiederum naturalisiert wurden. Auch die Nation als Ganzes wurde im ethno-nationalistischen Bild des Familienstaates als blutsverwandtschaftli- 
cher Organismus naturalisiert. Die von staatlicher Seite betriebene Formierung und Restrukturierung der nationalen Identität bediente sich auch hier ethno-nationalistischer Argumente und Bilder. Im Folgenden sollen diese Ansätze anhand prominenter Diskurse der Frauenbewegung überprüft werden. $\mathrm{Zu}$ fragen ist dabei, inwieweit Feministinnen ab der Taishō-Zeit (1912-1926) an unterschiedliche Instanzen der ethno-kulturellen Nation oder des politischen Staates appellierten und wie sie dabei ihre Forderungen nach Teilhabe jeweils unterschiedlich begründeten.

\section{FEMINISMUS, StAAT UND NATION IN DER VORKRIEGSZEIT}

Die Mutterschutz-Debatte (bosei hogo ronsō) $)^{4}$ von 1918/19 war die erste öffentliche Debatte in der japanischen Frauenbewegung um die politischen Voraussetzungen und die staatliche Verantwortung für den Schutz von Mutter und Kind. Hiratsuka Raichō (1886-1971), Übersetzerin von Ellen Keys Schriften, Herausgeberin der Seitō [Blaustrumpf], der ersten ausschließlich von Frauen verfassten literarischen Frauenzeitschrift Japans (Neuss 1971) und mithin die bekannteste japanische Feministin des 20. Jahrhunderts (Tomida 2004), forderte darin - wie Ellen Key in Schweden - angesichts steigender Sterblichkeitsraten erwerbstätiger junger Mütter (Kawai 1990) den rechtlichen Schutz und die staatliche Unterstützung für Frauen während der Schwangerschaft und Mutterschaft (Hiratsuka 1988a). Beeinflusst durch die nach dem Ersten Weltkrieg verstärkt geführte Menschen- und Völkerrechtsdiskussion betonte sie die Idee des Menschenrechtes für Frauen und Kinder (Yoneda 1990).

Die Dichterin Yosano Akiko (1878-1942) hielt diese Erwartungshaltung an den Staat und an die Männer für "parasitär" (kiseiteki). ${ }^{5}$ Sie vertrat die

4 Die wichtigsten Aufsätze finden sich in einer von Kōuchi (1988) herausgegebenen Quellensammlung. Zum Maternalismus Hiratsukas siehe Furukubo (1991); eine kritische Evaluierung von Hiratsukas und Ichikawa Fusaes maternalistischen Äußerungen während des Pazifischen Krieges liefert Suzuki (1995a).

5 Yosano (1988a: 83) schreibt: „Heutzutage paralysiert die bequeme Lebensweise solch parasitärer Frauen - eine grundlose, hässliche und feige Bequemlichkeit - im Innern den unabhängigen Geist der Frauen, belässt sie wie bisher in einem Zustand der Unwissenheit und Schwäche und lässt sie in Umständen verrotten, in denen sie halb Konkubinen, halb Dienerinnen sind; während im Außen die Männer nach wie vor auf die Frauen herabsehen und sich weigern, ihre tyrannische Haltung, die sie den Frauen gegenüber an den Tag legen, aufzugeben". Sicherlich geht es Yosano am Ende um eine gleichberechtigte Beteiligung von Mann und Frau am privaten und gesellschaftlichen Leben, ihre Kritik und ihr Aufruf richten sich jedoch in erster Linie an die Frauen selbst. 
individualistische Auffassung, dass Frauen erst dann Kinder bekommen sollten, wenn sie wirtschaftlich selbstständig wären (Yosano 1988b: 85). Den sozialstaatlichen Forderungen Hiratsukas und den auf die Unabhängigkeit von den Männern und vom Staat zielenden Vorstellungen Yosanos hielt die Sozialistin Yamakawa Kikue (1890-1980) entgegen, dass es diesen Ansätzen an einer grundlegenden Kapitalismuskritik mangele (Yamakawa 1988a:145) und der wirkliche Schutz des Existenzrechtes aller Menschen erst unter den veränderten Produktionsverhältnissen einer reformierten, und das heißt sozialistischen Gesellschaft gewährleistet wäre. ${ }^{6}$ Hier soll nicht die gesamte Debatte vorgestellt, sondern primär auf die Adressaten der feministischen Agenda hingewiesen werden. Unterschiedliche feministische Argumente zeigen, wie Frauen Mutterschaft mit gesellschaftlichen, kulturellen und staatlichen Vorstellungen verknüpften und sich so bewusst als geschlechtlich bedingte Subjekte auf den Staat bezogen oder sich von ihm distanzierten.

Hiratsukas Forderung nach Schutz wird auf der Grundlage der Postulierung eines geschlechtsspezifischen, gesellschaftlich und staatlich relevanten Beitrages von Frauen direkt an den Staat gestellt:

Was Kinder anbelangt, so sind auch die eigenen, von mir selbst geborenen Kinder nicht mein Privateigentum (shiyübutsu), sondern sind Sache (mono) dieser Gesellschaft und dieses Staates. Da die Anzahl und die Qualität der Kinder höchste Bedeutung für die fortschrittliche Entwicklung des Staates und der Gesellschaft und für deren zukünftiges Schicksal hat, ist die Aufgabe der Mutter, Kinder zu gebären und großzuziehen, bereits keine individuelle Aufgabe mehr, sondern eine gesellschaftliche und staatliche. (Hiratsuka 1988a: 108; vgl. auch Hiratsuka 1988b: 89)

Der eugenische Einschlag in Hiratsukas Argumentation ist auf den Einfluss von Keys Schriften zurückzuführen, die jedoch in eugenischer Hinsicht wesentlich weiter gehende Thesen verfocht und auch rassistische

6 Eine weitere an der Debatte beteiligte Person, Yamada Waka (1879-1957), verfocht in starker Anlehnung an Ellen Key den Mutterschutz und eine rechtliche Höherstellung der Frauen in der Familie. Sie begründete dies mit dem gesellschaftlichen Beitrag, den Frauen in Ausübung ihrer "Berufung" (tenshoku) als Hausfrau und Mutter leisteten (Yamada 1988: 95). Yamakawa liefert eine scharfe Kritik an den differenztheoretischen und zum Teil sozialdarwinistischen Theorien Keys und Yamadas. Sie nennt Key eine „Reaktionärin“ (Yamakawa 1988b: 118) und u.a. Yamadas Positionen einen "Verrat an den Frauen" (Yamakawa 1988b: 117). 
Ansichten hegte. ${ }^{7}$ Yosano Akiko, die selbst dreizehn Kinder auf die Welt brachte und elf davon großzog, antwortete in einer Replik an die Hauptbeteiligten der Debatte auf Hiratsukas Position:

Ich halte Kinder weder für "Sachen“ (mono) noch für "Mittel“ (dōgu). Ich halte sie für selbst- und eigenständige individuelle Charaktere. Kinder gehören nur sich selbst [...] sie sind kein Eigentum des Staates. (Yosano 1988c: 188)

Mit diesen und anderen Zitaten hat auch Kanō Mikiyo auf staatsbezogene feministische Äußerungen der Vorkriegszeit rekurriert und darauf hingewiesen, dass die Hauptargumentationen der Mutterschutzdebatte von europäischen politischen Strömungen beeinflusst waren: Yosano von der Frauenrechtsbewegung, Hiratsuka vom schwedischen Maternalismus und Yamakawa vom sozialistischen Feminismus (Kanō 2000: 104-05). Yamakawas sozialistische Position (siehe Kessler 1993) ist nur staatskritisch, insofern es sich um den bürgerlich-kapitalistischen Staat handelt. Während Hiratsuka ihre feministischen Forderungen an den Staat adressiert, lehnt Yosano jede staatliche Einmischung ab, auch wenn diese in geschlechtsspezifischen Sondermaßnahmen bestehen sollte. Ihr pazifistisches Gedicht zur Zeit des russisch-japanischen Krieges (1904/05), Kimi shini tamō koto nakare [Du darfst mir nicht sterben], ${ }^{8}$ in dem sie den Bruder vor dem Kriegsdienst und Soldatentod mit den Worten warnte: „Ah, mein Bruder, ich weine um dich, du darfst mir nicht sterben! [...] Ob sie fallen, die Wälle von Lüshun, oder nicht - was kümmert's?" (Schaarschmidt 1984: 60), steht in Einklang mit ihrer oben zitierten Weigerung, dem Staat ein legitimes Interesse an Kindern zuzugestehen. Ihre Aussagen postulieren die Priorität von Familienbindungen gegenüber staatlicher Inpflichtnahme.

7 So schrieb Key beispielsweise 1905: „Bis auf weiteres werden die Heime [= Familien, A.G.] [...] weder rezensiert noch preisgekrönt! Aber diese Zeit kommt vielleicht noch - so wie man schon jetzt in Frankreich das siebente Kind auf Staatskosten erzieht und Orden für die Frauen beantragt, die die größte Anzahl tüchtiger Kinder geboren und erzogen haben!“ (Key 1905: 264). In rassistischer Hinsicht argumentiert sie im selben Buch: „Die Liebesauswahl ist schon in gewissen Fällen - wie in Bezug auf nahe Blutsverwandtschaft, verschiedene Rassen und gewisse Krankheiten - Instinkt geworden [...]. Kein Verbot, nur alle Impulse des Blutes hindern die Amerikanerin, einen Neger oder Chinesen zu heiraten" (Key 1905: 157).

8 Der Abdruck des Gedichtes in der Zeitschrift Myōjō [Morgenstern] führte zum Verbot der betreffenden Ausgabe (Sept. 1904). Zu Yosano siehe May (1975), eine vollständige Übersetzung des Gedichtes findet sich bei Schaarschmidt (1984: 60-61). 
Eine weitere Position in dieser Reihe nimmt Takamure Itsues (18941964) in den 1920er Jahren entwickelte Kritik an Ellen Keys Theorien ein. In ihrer 1926 veröffentlichten Schrift Ren'ai sōsei [Genesis der Liebe] formulierte Takamure ihr Konzept der Mutterliebe (boseiai), die sie als mütterlichen Instinkt mit der Natur in eins setzte und damit jegliche staatliche Intervention oder Vereinnahmung des Mütterlichen kritisierte (Germer 1996, Yamashita 1988). Gegen die Eugenik Ellen Keys gerichtet, die eine staatliche Preisverleihung für die Aufzucht "tüchtiger Kinder" (Key 1905: 264) propagierte, schrieb sie:

Gibt es in Bezug auf die Mütterlichkeit etwas Störenderes als die Inspektion und Preisvergabe von Seiten des Staates? [...] Das gesellschaftliche Bewusstsein, das die [...] untüchtigen Kinder diskriminiert, ist für die Mütterlichkeit das, was sie am meisten erschaudern lassen müsste. (Takamure 1926a: 109)

Naturgemäße Mütterlichkeit beinhaltet also Liebe zum Leben und eine Liebe, die alle Kinder umfasst. In anarchistischer Manier lehnt sie jede staatliche Einmischung in die Erziehung der Kinder als Herrschaftseingriff ab. Die Teilerfolge der bestehenden westlichen und japanischen Frauenbewegungen, die dem Staat bestimmte Rechte abtrotzen konnten, stellen sie nicht zufrieden, da ihr an einer umfassenden ästhetischen Erneuerung der Geschlechterordnung gelegen ist: „Auch wenn wir Rechte erwerben, bleibt unser Leben pervertiert. Auch wenn wir die Freiheit der Bildung erlangen, können wir über diese Bildung nicht selbst bestimmen" (Takamure 1926a: 111). In ihrer Kritik rekurriert sie immer wieder auf die Natur und natürliche Beziehungen. Eine in der Natur des Menschen angelegte Familie ist es, die die patriarchale Familie (ie) in Zukunft ersetzen soll:

Wir werden in Zukunft wohl immer mehr die Natur als unser Haus (ie) betrachten. Und das Wort von der Vergesellschaftung der Familie werden wir im Sinne einer Familiarisierung der Gesellschaft erfahren. (Takamure 1926a: 111)

Die Antithese zur staatlichen, zentralistisch-bürokratischen Bewältigung sozialer Aufgaben besteht in der Hinwendung zu einem natürlichen und autonomen Lebensvollzug einer als grundsätzlich egalitär imaginierten japanischen Gemeinschaft. Hierin liege der Beitrag, den gerade die japanischen Frauen, die „auf dieser Welt einzigartigen, weisen Frauen“ für die Entwicklung einer neuen Emanzipationsidee leisten können: In der mütterlichen Liebesfähigkeit liege die gesellschaftliche Stärke der Frauen, die Appelle an und Bestimmungen durch den Staat (sei es der japanische oder der sowjetische) obsolet erscheinen lässt (Takamure 1926a: 108, 213). 
Takamures staatskritische Haltung in den 1920er Jahren wird deutlich in ihrer aktiven Mitarbeit in anarchistisch orientierten Bauernvereinigungen und in ihrer Herausgeberschaft von Fujin sensen [Frauenfront], der ersten anarchistisch-feministischen Frauenzeitschrift in Japan (Nishikawa 1975, Tsurumi 1985, Germer 2000) von 1930 bis 1931. In der Folge und bis zu ihrem Tod 1964 wandte sie sich ganz der Erforschung der japanischen Frauengeschichte $\mathrm{zu}$, als deren Pionierin sie heute bekannt ist. Ihr kultureller Nationalismus in den 1930er Jahren und ihre Beiträge zu Organen der Kriegsregierung in den 1940er Jahren, um die es u.a. im Folgenden gehen soll, werden verständlicher, wenn wir ihre und die Positionen anderer Feministinnen in Bezug auf die Adressaten ihrer Kritik bzw. ihrer Appelle unterscheiden.

Hier kann Doaks oben genannte Analyse des ethnischen und staatskritischen Nationalismus in Japan (Doak 1996, 1997) auch für die feministische Geistesgeschichte fruchtbar gemacht werden. Takamure und Yosano setzten in ihrer Staatskritik Familien- bzw. Gemeinschaftsbeziehungen einem anonymen staatlichen (westlichen, männlich konnotierten, marxistischen) Überbau entgegen. Demgegenüber appellierten, wie weiter unten zu zeigen ist, Feministinnen wie die bereits genannte Maternalistin Hiratsuka Raichō, aber auch egalitär argumentierende Feministinnen wie die Vorsitzende des Bundes für Frauenwahlrecht, Ichikawa Fusae (1893-1981), die Ärztin und Schulleiterin Yoshioka Yayoi (1871-1959) oder die Internationalistin und Universitätsdirektorin Inoue Hideko (1875-1963), die zum Teil kooperierten und während der Kriegszeit politische Ämter innehatten, in ihren Forderungen schon immer an das Parlament oder an den Staat mit Argumenten, die den Beitrag von Frauen auch im öffentlichen, männlich bestimmten Raum hervorhoben.

\section{FEMINISMUS UND DER NATIONALSTAAT IN DER KRIEGSZEIT}

Die nationalstaatliche Innen- und Außenpolitik Japans war in den 1930er und 1940er Jahren gekennzeichnet durch zunehmende Militarisierung, internationale Isolation durch den Austritt aus dem Völkerbund (1933), rhetorische Einbindung des Volkes unter der Vorgabe eines Familienstaates, der auch ethnische Differenzen überbrückte (Oguma 2002), und staatliche Repression divergierender politischer Kräfte. Im Jahr 1931 markierte der japanische Einfall in die Mandschurei den Beginn des im Japanischen sogenannten 15jährigen Krieges. 1937 erschien die zentrale ideologische Publikation des japanischen Nationalstaates, Kokutai no hongi [Essenz des 
nationalen Wesens $],{ }^{9}$ in der im Rahmen des Familienstaates der Frau in der Familie eine dem Soldaten, der in den heiligen Krieg zieht, analoge Rolle zugeschrieben wurde.

Im Gegensatz zur Meiji-staatlichen Geschlechterideologie, in der die Position des Vaters zentral war (unterstützt durch das Meiji-Zivilrecht), rückt, wie Kano Masanao (1983) gezeigt hat, während des Krieges Mutterschaft im Interesse des Staates (kokkateki bosei) und das Image der Wärme und Fruchtbarkeit in der Familie in den Mittelpunkt (vgl. auch Miyake 1991: 271). In zahlreichen Publikationen wird die japanische Mutter (Nippon no haha) gefeiert, beschrieben und erfunden. ${ }^{10}$ Flankiert waren diese ideologischen Instrumentalisierungen, die im Slogan umeyo fuyaseyo [Gebärt und vermehrt euch] ihren pointierten Ausdruck fanden, von sozialer Gesetzgebung. Zentral war hier die erstmalige Mutterschutzgesetzgebung von 1937 (Boshi hogo hō), die seit 1934 von Feministinnen gefordert worden war. 1941 trat das nationale Eugenik-Gesetz (Kokumin yūsei hō) in Kraft, Ausdruck der pronatalistischen und eugenischen Politik des Regimes. Der während des 15jährigen Krieges auf gesellschaftlicher wie auf staatlicher Ebene vorangetriebene Diskurs der Mutterverherrlichung fügte sich Kanō Mikiyo (1989: 69) zufolge in die pronatalistische Politik der „Stärkung und Vermehrung der ,menschlichen Ressourcen' für den Invasionskrieg" ein und war deshalb - in diesem Ausmaß erstmalig in der japanischen Geschichte - von sozialpolitischen Mutter- und Kinderschutzmaßnahmen begleitet.

Feministische Reaktionen auf die Kriegs- und Expansionspolitik des japanischen Militärs und zum Kriegskurs der Regierung seit 1931 waren zunächst vielfältig. Die Frauenrechtlerin und Vorsitzende des Bundes für Frauenwahlrecht (Fujin Sanseiken Kakutoku Dōmei), Ichikawa Fusae, rief

9 Erstmals im März 1937 herausgegeben und an das Lehrpersonal des Landes verschickt, wurde dieser in schwer verständlicher klassischer Schriftsprache verfasste Text in verschiedenen Ausgaben millionenfach aufgelegt. Dieses politische Traktat stellte die offizielle Interpretation der fundamentalen Prinzipien des "nationalen Wesens" (kokutai) dar. Es stellte das Göttliche von Ursprung, Führung und Eigenheiten Japans als Ursache für die Prosperität des Landes heraus und beschrieb Sprache, Sitten, Gebräuche, Religionen, das politische System der Meiji-Verfassung und die direkte Herrschaft des Kaisers mit dem Ziel, Japans göttliche Mission zu illustrieren (Gauntlett und Hall 1949, Tsunoda et al. 1958).

10 Vgl. Kanō (1989: 75). Unter den namhaften SchriftstellerInnen und Intellektuellen, die sich Anfang der 1940er Jahre in einer Anzahl von Publikationen wie etwa Nippon no haha [Die Mütter Japans] von 1942 dem Thema der ,japanischen Mutter" näherten, befand sich neben Yanagita Kunio, Ishida Eiichirō, Kawabata Yasunari, Takamura Kōtarō, Ozaki Ichio und Satō Haruo auch Takamure Itsue. 
1931 zur Bewusstwerdung der Frauen als „Mütter der Menschheit" und zur Zusammenarbeit von Frauen in Japan und China auf, um zu einer schnellen Lösung und Beendigung des Konfliktes zu kommen (Kanō 2000: 106). Widerstand gegen die staatliche Expansionspolitik wurde auf dem jährlich stattfindenden Frauenwahlrechtskongress noch 1934 deutlich von der Position als Mütter aus formuliert. Allmählich jedoch weichte die Haltung auf, und die im Zusammenhang mit der nationalen Mobilisierung 1937 gegründete Nachfolgeorganisation, der Bund japanischer Frauenvereinigungen (Nippon Fujin Dantai Renmei), propagierte die Kriegsmobilisierung der Frauen in Slogans wie „Kein Aufbau ohne Frauen!“ (josei no kyōryoku nakushite kensetsu nashi) (Nishikawa 1990: 236). Der Kampf um das Frauenwahlrecht wurde bewusst aufgegeben. Der Appell an den Staat und der Kampf um das damit verbundene Recht auf Teilhabe an der politischen Gesellschaft und auf Teilnahme an politischen Entscheidungsfindungen in nationalen und internationalen Belangen wurde jedoch beibehalten in dem Versuch, durch die Bekleidung öffentlicher Ämter in der Kriegsregierung im vorgegebenen Rahmen Einfluss zu nehmen. Hierzu gehören viele der bekanntesten Feministinnen der Vorkriegszeit wie Ichikawa Fusae, Kōra Tomiko (1896-1993) oder Inoue Hideko. $^{11}$

Ein weiteres Beispiel ist die während des Pazifischen Krieges politisch aktive Ärztin Yoshioka Yayoi, die bereits 1900 eine Medizinfachschule für Frauen gegründet hatte. Ihre Argumentation für Bildung und Berufstätigkeit der Frau war stets mit dem Dienst der Frauen für den Staat verknüpft. 1939 reiste sie zu Studienzwecken u.a. nach Deutschland, von wo sie das Konzept der nationalsozialistischen Gleichschaltung aller Frauenorganisationen nach Japan brachte und vehement vertrat. Sie war die einzige Frau, die von der Kriegsregierung in das Leitungsgremium der Zentralen Liga für Nationale Geistige Mobilisierung (Kokumin Seishin Sōdōin Chūō Renmei) berufen wurde (Nishikawa 1990: 245).${ }^{12}$ Doch Hunderte weiterer Frauen wurden in den Mobilisierungskomitees unterschiedlicher Ministerien eingesetzt. Nishikawa (1997: 59) schreibt hierzu: „Never before had Japanese women attained visibility in public life in such numbers“.

$11 \mathrm{Zu}$ den diversen Regierungsposten dieser Feministinnen siehe Katzoff (2000: 204-06) und Nishikawa (1997). Vor allem Ichikawa hatte ab 1937 zahlreiche Ämter inne. Zur Internationalistin Inoue, die sich schließlich dem Pan-Asianismus verschrieb, siehe Schneider (2007).

12 Nach dem Krieg wurde Yoshioka aus ihrem Amt als Schulleiterin entfernt, erhielt jedoch bereits 1955 die höchste staatliche Auszeichnung für Frauen (fujin bunka shō) (Mitsui 1963). 
Bei den staatskritischen Feministinnen hingegen verlief die Hinwendung zum Nationalstaat über die Verehrung des Kaiserhauses als Symbol der ethnischen und kulturellen Affirmation der Nation und über die Imagination des Volkes als einer auf quasi natürlichen, blutsverwandtschaftlichen Bindungen beruhenden Familie. Die Pazifistin Yosano Akiko, die in der Mutterschutzdebatte von 1918 eine anti-maternalistische sowie bis zu Beginn der Shōwa-Zeit eine dezidiert geschlechtsegalitäre, dabei aber staatsferne Position vertrat und sich zudem als Teil des Proletariats verstand, veröffentlichte bereits 1927 ein Gedicht, in welchem sie dem tennō huldigte. Sie verteidigte den Einfall in die Mandschurei 1931 aus nationalistischer und pan-asiatischer Sicht, wenngleich sie die Gewalt kritisierte, und unterstützte ab 1932 durchgängig den kriegspolitischen Kurs der Regierung (Nagahara 1988: 52-53, Katzoff 2000: 88-89).

Auch in Deutschland war die Idee der Nation im 19. Jahrhundert mit der Rekonstituierung des organizistisch-politischen Modells einhergegangen, das sein Vorbild nicht im Vertragsrecht, sondern in Familienbeziehungen und seinen spezifischen Abhängigkeiten, Pflichten und Verantwortlichkeiten fand. Die Ausweitung des Begriffes Familie zur nationalen Familie bot auch hier den Frauen Argumente für die Forderung nach Erweiterung der Handlungsspielräume im öffentlichen Raum. Die Rolle der Frau als Bürgerin blieb aber dadurch auch geschlechtsspezifisch geprägt und beschränkt (Blättler 2000: 114). Bei der staatskritischen, anarchistischen Feministin Takamure nahm das Konzept der Mütterlichkeit die Form eines nationalen Mythos an und wurde von ihr während des Pazifischen Krieges in einem Aufruf zum Patriotismus zur ursprünglichen Blutsliebe des Mutterherzens (hahagokoro) stilisiert (Takamure 1944a). ${ }^{13}$ Je mehr die Nation von staatlicher Seite im Bild der Familie beschrieben und auf dieser natürlichen Grundlage propagiert wurde, umso widerspruchsfreier konnte die patriotische Integration der staatskritischen Frauen in den Nationalstaat erfolgen.

Bereits seit 1925 hatte Takamure (besonders 1926a) immer wieder ihre Moderne-, Kultur- und Staatskritik mit einer Kritik am Westen und westlichen Philosophen mit der Überzeugung verbunden, „dass das Morgengrauen unseres ,Neuen Orientalismus' (shin Tōyō-shugi) unbedingt aus unserem Land der Ahnen hervorgehen wird" (1925, zit. nach Koyama 1982: 105). Kano und Horiba (1985) reihen Takamure in die Tradition japanischer Intellektueller ein, die während des Zweiten Weltkrieges die geis-

13 Vgl. im Folgenden Germer (2003: 46-49). In Takamures Anschauung war das Mutterherz in seiner allumfassenden Liebe mit dem göttlichen Herzen (kamigokoro) und letztlich auch mit dem kaiserlichen Herzen (ōmikokoro) synonym (Takamure 1944a; vgl. Kanō 1989: 69). 
tige Rückkehr nach Japan (Nippon e no kaiki) und die „Überwindung der Moderne" (kindai no chōkoku) propagierten. ${ }^{14} 1934$ rechtfertigte Takamure den Einfall in die Mandschurei (1931) als Akt des Widerstandes gegen eine internationale Ordnung, die von den fortgeschrittenen westlichen Nationen im 19. Jahrhundert auf gewaltsame Weise geschaffen worden sei und die im 20. Jahrhundert von einer Institution wie dem Völkerbund konserviert werden solle (Takamure 1934: 207; vgl. Germer 1996: 172-73). Sie sprach in diesem Zusammenhang vom "Volksgeist Japans" (Nippon no minzoku seishin) und seiner Aufgabe in Asien und benannte damit die Ideologeme Nipponismus und Asianismus, die Hauptpfeiler der Ideologie der "Großostasiatischen Wohlstandssphäre" (Dai Tōa kyōeiken) (Kojima 1993). 1937 rief sie erstmals die Frauen dazu auf, sich in Krisenzeiten innerhalb des Systems zu engagieren (Takamure 1937) und 1940 veröffentlichte sie ihre Schrift Josei 2600 nenshi [2600jährige Geschichte der Frau] ${ }^{15}$ und leistete damit einen Beitrag zu der Regierungskampagne, die jenes Jahr zum nationalen Jubiläumsjahr der legendären Reichsgründung vor 2600 Jahren machte mit dem Ziel, die Loyalität und die Vorstellung des japanischen Volkes als Schicksalsgemeinschaft im Krieg zu stärken. In dieser Zeit erschienen zahlreiche solcher Historien - im Jahr zuvor war

14 Im Jahre 1942 fand unter der Teilnahme verschiedener Intellektueller eine von der Zeitschrift Bungakkai veranstaltete Konferenz unter dem Motto „Die Überwindung der Moderne" (Kindai no chōkoku) statt, deren Symposiumsberichte in den September- und Oktobernummern erschienen und 1943 als Monographie unter dem gleichnamigen Titel veröffentlicht wurden (Chiteki Kyōryoku Kaigi 1943). Auch wenn dieser bereits früher von Takayama Chogyū geprägte Ausdruck kein einheitliches Gedankengut bezeichnet und die Konferenzteilnehmer sehr unterschiedliche Meinungen vertraten, wurde der Titel zum Slogan und zu einem Symbol für die intellektuelle Kooperation im Faschismus (Takeuchi 1987).

15 Wie Takamure im Vorwort schreibt, wurde die Schrift in der vom Zeitungsverlag Asahi herausgegebenen Neujahrsnummer Fujin Asahi 1940 erstmals veröffentlicht und mit einigen Ergänzungen und einem autobiographischen $\mathrm{Ab}$ schnitt versehen als Monographie im Februar des selben Jahres im Verlag Kōseikaku aufgelegt. Vom Zeitungsverlag gebeten, habe sie nur zwei Wochen zum Verfassen der Schrift gehabt, die Chance jedoch aufgrund des historisch einmaligen Datums und der Möglichkeit, die Themen Frauengeschichte und Frauenfragen einzubringen, ergriffen (Takamure 1940: Vorwort). Die Kernthesen besagen, dass Mann und Frau, in der Frühzeit aufeinander angewiesen, weitgehend gleichgestellt gewesen seien, und auch Frauen einen wesentlichen Beitrag zur Reichsgründung und zur Entwicklung der Kultur geleistet hätten. Die gesellschaftliche Entwicklung habe jedoch allmählich, und verstärkt seit der Tokugawa-Zeit (1600-1867), zu einer Verschlechterung der Stellung der Frauen geführt, welche trotz vereinter Bemühungen fortschrittlich gesinnter Männer und Frauen bis in ihre Zeit anhalte (Takamure 1940: 122-24). 
bereits die „2600jährige Geschichte Japans“ (Nippon 2600 nenshi) von Ōkawa Shūmei (1886-1957), einem der bekanntesten Ideologen der politischen Rechten zwischen 1920 und 1945, erschienen (Ōkawa 1939).

\section{Die Grossjapanische FraUENVEREINIGUNG \\ (DAI NiPPON FUנINKAI)}

Mit der Gründung der Großjapanischen Frauenvereinigung (Dai Nippon Fujinkai, kurz: Nippu) am 2. Februar 1942 kamen Staat, Nation und Feminismus in institutioneller Form zusammen (Katzoff 2000: 149-209). Die Neugründung war eine Maßnahme der Taisei Yokusankai [Imperial Rule Assistance Association], welche sich am deutschen Vorbild der Gleichschaltung orientierte. Ihr war die Auflösung dreier bis dahin existierender Frauenorganisationen vorausgegangen: Aikoku Fujinkai [Patriotische Frauenvereinigung, seit 1901], Dai Nippon Kokubō Fujinkai [Großjapanische Frauenvereinigung zur Landesverteidigung, seit 1932] und Dai Nippon Rengō Fujinkai [Großjapanischer Bund der Frauenvereinigungen, seit 1931]. Diese Vereinigungen hatten Frauen in unterschiedlichen Schichten repräsentiert, waren aber zum Teil mit ähnlichen Aufgaben befasst und konkurrierten miteinander.

Die von Männern geführte vereinigte Organisation hatte nach eigenen Angaben mehr als 27 Millionen Mitglieder, inklusive der sieben Millionen in den von Japan besetzten oder kolonisierten Gebieten Korea, Taiwan, Sachalin und die Südsee-Territorien (Suzuki 1995b, Katzoff 2000: 195, Wilson 2006: 215). Ziel der gleichgeschalteten Vereinigung war eine zentralisierte und damit besser kontrollierbare Organisation, die aber nach außen hin von Frauen selbst für die Verbesserung der Lebensbedingungen von Frauen als effektiver und durchsetzungskräftiger begrüßt wurde. Die Kooperation mit der Kriegsregierung zielte auf Reform durch Partizipation (Katzoff 2000: 152). Die Nippu-Mitglieder betonten die Geschlechterdifferenz und erklärten, dass sie ihr Frausein in den Dienst der Nation stellten. Dabei bildete dieses Frausein keineswegs eine auf Kinder und Küche beschränkte Weiblichkeit ab.

Das Organ der Vereinigung, die Zeitschrift Nippon Fujin [Die Japanische Frau], war ehedem die offizielle Zeitschrift der Kokubō Fujinkai und, nachdem die Regierung 1941 die meisten anderen Frauenzeitschriften zur Einstellung gezwungen hatte, die wesentliche Informationsquelle für Frauen. Sie bietet reichhaltiges Anschauungsmaterial für die Fülle an unterschiedlichen Berufen und zum Teil widersprüchlichen Weiblichkeitsbildern, die nur in einer Hinsicht eindimensional waren: in ihrer Propagierung einer moralisch hochstehenden Weiblichkeit und ihrer unbeding- 
ten Ausrichtung auf den Nationalstaat. Auf unterschiedlichste Weise wurden Identifikationsangebote unterbreitet, die Frauen dazu mobilisieren sollten, sich für die Nation zu engagieren. Regelmäßige bildliche Darstellungen der Kaiserin, die die Präsidentin der Großjapanischen Frauenvereinigung war, sowie Ansprachen und Botschaften Tōjō Hidekis und von Leitern verschiedener Ministerien bildeten den politischen Rahmen und betonten auch inhaltlich die eminente staatstragende Bedeutung des Beitrages von Frauen für die Nation. Dabei führte, wie Wilson (2006) argumentiert, die starke Betonung der geschlechtsspezifischen Rollenverteilung und der Verantwortung der Frauen für Heim und Familie einerseits, sowie staatliche und kriegsbedingte Notwendigkeiten der Arbeit von Frauen außer Haus andererseits zu Spannungen und Loyalitätskonflikten, die sich mit Fortschreiten des Krieges verschärften und durch eine immer stärker werdende ideologische Rhetorik des Familienstaates überdeckt werden mussten.

Zahlreiche Aktivistinnen der Frauenbewegung der Vorkriegszeit engagierten sich in der Organisation und kamen in Nippon Fujin zu Wort. Allein in der ersten Ausgabe finden sich die Beiträge und Namen von Hani Setsuko, Yoshiya Nobuko, Yoshioka Yayoi, Kawasaki Natsu, Hoshino Tachiko, Ōhama Hideko, Nogami Yaeko und Takamure Itsue. Weitere Feministinnen wie Yamataka Shigeri und Kōra Tomiko tauchen in späteren Ausgaben auf. Von 1942 bis kurz vor Kriegsende lieferte Takamure insgesamt dreiundzwanzig Beiträge in dieser Zeitschrift. 1943 hob sie in dem Artikel „Gunji to josei“ [Das Militär und die Frauen] die in der Mythologie überlieferten kriegerisch-kämpferischen Fähigkeiten japanischer Frauen hervor, um sie im Kampf gegen die westliche Bedrohung zu reaktivieren (Takamure 1943, vgl. Germer 1996: 172-73). Mit Fortschreiten des Krieges wurde auch ihre Rhetorik schärfer und 1944 schrieb sie in Nippon Fujin:

Es war wohl zur Zeit des russisch-japanischen Krieges, als Frauen Zeilen schrieben wie „Bruder, Du darfst mir nicht sterben“ oder „im Herzen einer Frau gibt es keine Hauptstadt". Wenn Friedenszeiten wären, könnte man dieses Gejammere irgendwie entschuldigen, aber in der Notlage, in der sich das Land jetzt befindet, ist das anhaltende Jammern einer japanischen Frau nicht würdig. [...] Im Ausdruck „,im Herzen einer Frau gibt es keine Hauptstadt" etwa wird das „Herz einer Frau“ als Gegensatz zum "öffentlichen Herzen (ōyake no kokoro)" definiert und dies weicht völlig von dem ab, was die japanische Frau ausmacht. Die Redeweisen „weil sie eine Frau ist" oder "das Gemüt einer Frau“ (onnagokoro) sind in ihrem herkömmlichen Sinn heutzutage vollkommen zurückzuweisen. (Takamure 1944b: 11) 
Dass die Identifikation der Frau mit ihrer Nation und einem nationalen Geschlechtscharakter die Möglichkeit der Selbstaffirmierung mit sich führt, die den Konflikt zwischen den Geschlechtern verdeckt (Blättler 2000: 116), wird in obiger Aussage besonders deutlich. In den letzten Worten desselben Artikels bringt Takamure ihre Aussagen noch einmal auf den Punkt: „Das Öffentliche und das Private sind eins (kōshi ichinyo)“ (Takamure 1944b: 11). Dieses Postulat, das Takamure bereits seit Mitte der 1920er Jahre staats- und gesellschaftskritisch gegen die Unterordnung der privaten Sphäre (der Frauen) unter die öffentliche Sphäre (der Männer) vorgebracht hatte (Takamure 1926b, vgl. Germer 1996: 137), wendet sich hier ins Totalitäre: Staat und Nation, das Öffentliche und das Private, Männer und Frauen, das Individuum und die Gesellschaft stellen keine konflikthaften Beziehungen mehr dar, sondern werden, angesichts des Feindes und der nationalen Notlage, eins im Sinne einer Familie.

Eine ähnliche Ineinssetzung von Staat und Nation hatte 1943 der Direktor der Vereinigung, Kawanishi Jitsuzō (1889-1978), in den folgenden Worten formuliert: „Ist es nicht so, dass die Einheit, die wir einhundert Millionen, die unter dem Kaiser leben, bilden, der japanische Staat ( $k o k k a)$ ist? So etwas wie einen Staat, der irgendwo anders, getrennt von jedem individuellen Mitglied der Nation (kokumin), existiert, gibt es nicht ${ }^{\prime \prime}{ }^{16}$ Auch seine weiteren Ausführungen zielen darauf ab, dass kein Unterschied bestehe zwischen Regierung, Volk und individuellem Schicksal und Wollen.

Das ideologische Bindeglied zwischen Individuum und politischem Staat (Regierung) liegt in der ethnischen Idee der Nation, vermittelt über die Ideologie der Familie. Dass die Familie darüber hinaus ideologisch auch den expansiven Nationalstaat rechtfertigen kann, zeigt Takamure in ihrer Definition von „Familiengesinnung" und der Rolle der Frauen darin in einem anderen, ebenfalls in Nippon Fujin 1944 erschienenen Artikel:

Unter Familiengesinnung verstehe ich ein blutsverwandtschaftliches Gefühl, das sich von Geschwistern und Eltern auf die ganze Menschheit ausweitet. [...] Unser weibliches Prinzip (taoyame) ist das Lebenselixier unserer Familiengesinnung und ist von dem Wunsch beseelt, dass die ganze Welt eine Familie werde. Da nun unser heiliger Krieg gegenüber denjenigen begonnen wurde, die diese Familienwerdung

16 Kawanishi (1943), in Suzuki (1995b: 448), zit. bei Wilson (2006: 226). Anders als die Inhaberinnen der zeremoniellen Ämter der Organisation waren die wirklichen Führer Männer. Kawanishi war ein früherer Beamter des Innenministeriums und früherer Gouverneur der Präfekturen Saitama, Nagasaki, Kioto und Tokio. 
behindern, kann man wohl sagen, dass der Krieg auch eine aktive Sache der Frauen ist. (Takamure 1944c: 8-11)

Die Maternalistin Takamure befürwortet den Krieg und die Expansion auf der Grundlage der Teilnahme der Frauen, die ihre Stärken und Kräfte gesellschaftlich in einem System einsetzen können und wollen, in dem der Staat und die weiblich konnotierte Nation als Familie verschmolzen sind. Das Konzept der Familie ist hier nur ein Element der umfassenderen Argumentation, in der es um die Teilhabe geht, die den Frauen in der Kriegssituation und an der Rettung der Welt zukommt, ein Beitrag, um den von staatlicher Seite aktiv geworben und der andererseits eingefordert wird. Mit dieser Teilhabe gewinnt die Frau gleichzeitig eine Position der missionarischen Überlegenheit nicht nur gegenüber den asiatischen Geschwistern und den westlichen Feinden, sondern auch gegenüber den japanischen Männern.

Die Aussicht auf Teilhabe an gesellschaftlicher und politischer Macht, an Bürgerrechten ${ }^{17}$ im Nationalstaat war der Motor der Kooperation sowohl für staatsbejahende als auch für staatskritische Feministinnen, für ausgesprochene Maternalistinnen und gleichermaßen für Frauen geschlechtsegalitärer Ausrichtungen innerhalb der japanischen Frauenbewegung seit der Vorkriegszeit. Sie folgten schließlich der Regierung auf ihrem imperialistischen und kaisertreuen Kurs oder spielten eine aktive weibliche Rolle darin. Wie Garon, Havens und Katzoff vertrete ich die These, dass es sich dabei sowohl von staatlicher als auch von feministischer Seite nur bedingt um einen Strategiewechsel gehandelt hat. Garon (1993) arbeitet in seiner Forschung eine Kontinuität in der politischen Integration von Frauengruppen im japanischen Staat von 1890 bis 1945 heraus und Katzoff (2000) beschreibt den pragmatischen Umgang von Frauengruppen und Feministinnen mit staatlichen Vorgaben und Spielräumen während des Asiatisch-Pazifischen Krieges. Eine vergleichsweise verhaltene staatliche Nutzung der Ressource weiblicher Arbeitskraft in der japanischen Kriegsindustrie (Havens 1975) führt Miyake (1991) auf die spezifische Kombination von ideologischen, sozialökonomischen und kriegswirtschaftlichen Erwartungen an Frauen zurück.

17 Der Begriff Bürgerrechte ist zwar mit Rechten von BürgerInnen in einer vom Staat separierten Öffentlichkeit konnotiert, weshalb er sich natürlich nicht zur Beschreibung eines Volkes von Untertanen (shinmin) im tennō-System, das den Kaiser als Souverän setzt, eignet. Ich verwende den Begriff hier jedoch als zusammenfassende Utopie der Frauenbewegung, deren Strategien auf die Teilhabe an gesellschaftlicher und politischer Macht als gleichberechtigte Bürgerinnen abzielten. 


\section{SCHLUSSFOLGERUNGEN}

Die von staatlicher Seite forcierte Ineinssetzung und Naturalisierung von ethnischer Nation und Staat mittels einer Ideologie des Familienstaates ermöglichte es ehemals staatskritischen bzw. -fernen Feministinnen, auch die Logik des Staatsapparates zu akzeptieren, und ehemals an staatliche Institutionen appellierende Feministinnen, auch die ethno-nationalistische und differenztheoretische Rhetorik des Nationalstaates zu übernehmen. Doch sollte dies nicht dem Aufgeben eigener ursprünglicher emanzipatorischer Motivationen gleichgesetzt werden. Die Ausweitung der Einflusssphäre unter Rekurs auf eine radikal differente und moralisch integre Weiblichkeit ist eine Bewegung der originären Selbstbehauptung, deren Adressaten sowohl die dominierenden Männer als auch die japanischen Frauen sind. Die Verbindung zwischen Vorkriegs- und Kriegs-Feminismus in Japan liegt in der Forderung nach Bürgerrecht, die sich nicht auf die an den Staat adressierte Forderung nach Wahlrecht beschränkt, sondern in einem weiteren Sinne zu verstehen ist: als Zielsetzung auf die volle Teilhabe an der kulturellen, gesellschaftlichen und politischen Gestaltung der Lebenswelt als geschlechtlich bedingte Subjekte.

Von Seiten des Staates wurde deutlich, dass im männlich dominierten Prozess der Nationsbildung Frauen, Familie und geschlechtliche Repräsentationen wichtige Elemente darstellen, die sowohl auf die Strukturierung im Inneren als auch auf die Abgrenzung nach außen abzielen. Anderson (1998) hat in seiner für die Nationsbildung maßgeblichen Studie über „erfundene Gemeinschaften“ auf die Funktion von Geschlecht nur im Hinblick auf die Fraternisierung der Brüder hingewiesen. Die feministische Forschung nach ihm hat gezeigt, dass die Imagination und Reproduktion eines National- und eines Geschlechtscharakters nicht nur gleichzeitig entstanden, sondern zur wechselseitigen Repräsentation unerlässlich sind. Yuval-Davis schreibt über den vergeschlechtlichten kulturellen Diskurs:

In [...] culturalized discourse, gendered bodies and sexuality play pivotal roles as territories, markers and reproducers of the narratives of nations and other collectivities. [...] gender relations are at the heart of cultural constructions of social identities and collectivities as well as in most cultural conflicts and contestations. (Yuval-Davis 1997: 39)

Dies gilt für die emanzipatorische feministische ebenso wie für die fundamentalistische Narration. Frauen und Männer fügen sich im Prozess der internationalen Konkurrenz auf unterschiedliche Weise in das Projekt der Nationsbildung ein, wobei Frauen unterschiedliche und widersprüchliche Identifikationsmuster angeboten werden. 
Die politische und gesellschaftliche Entwicklung von Nation und Staat seit der Meiji-Zeit hin zu einem Nationalstaat absorbierte den ehemals staatskritischen, ethnischen und kulturellen Nationalismus (Doak 1996, 1997). In diesem Zusammenhang wurde Frauen sowohl eine geschlechtlich bedingte eigenständige kulturelle Rolle in der Nationsbildung zuerkannt, als auch von staatlicher Seite auf geschlechtliche Forderungen in Form von Schutzmaßnahmen reagiert. Dies bildet den Hintergrund dafür, dass sowohl Feministinnen, die in der Vorkriegszeit zur Verwirklichung ihrer emanzipatorischen Ziele, etwa soziale Rechte und Pflichten (Wohlfahrt) oder politische Rechte (Wahlrecht), an den Staat appellierten (Hiratsuka, Ichikawa, Yoshioka, Inoue), als auch staatskritische (bzw. -ferne) Feministinnen, die sich auf die Eigenständigkeit von Frauen in kultureller und sozialer Hinsicht beriefen (Takamure, Yosano), in der von zunehmender Repression gekennzeichneten politischen Situation der 1930er und 1940er Jahre den nationalstaatlichen Angeboten und Forderungen ,erlagen' und in dem ihnen gesteckten Rahmen des expansiven Nationalstaats ihre ursprünglichen Interessen aktiv zu verwirklichen suchten. Dieser wies den japanischen Frauen eine Subjekt-Position zu, die zwar beschränkt war, jedoch auch für die Frauen eine Position der Überlegenheit imaginierte - nicht nur gegenüber dem Westen, sondern auch gegenüber den ,zu erziehenden' Frauen Asiens. ${ }^{18}$

\section{LITERATURVERZEICHNIS}

Anderson, Benedict (1998): Die Erfindung der Nation: Zur Karriere eines folgenreichen Konzeptes. Berlin: Ullstein.

Anthias, Floya und Nira Yuval-Davis (Hg.) (1992): Racialized Boundaries: Race, Nation, Gender, Colour and Class and the Anti-Racist Struggle. London und New York: Routledge.

Blättler, Sidonia (2000): Nation und Geschlecht im philosophischen Diskurs der politischen Moderne. In: Feministische Studien 2, S. 109-18.

Blättler, Sidonia (2002): Nationale Identität, nationaler Gegensatz und die Geschlechterdifferenz am Beispiel von Fichtes „Reden an die deutsche Nation“. IWM Working Papers 2/2002. Wien: Institut für die Wissenschaften vom Menschen.

Bock, Gisela (1989): Frauen und der Nationalsozialismus: Bemerkungen

18 Solch eine erzieherische Haltung gegenüber chinesischen Frauen nahm beispielsweise Ichikawa Fusae ein. Sie verfocht die Idee der Großostasiatischen Wohlstandssphäre, in der sie ebenso wie Takamure den japanischen Frauen eine führende Rolle zusprach (Katzoff 2000: 109-10). 
zu einem Buch von Claudia Koonz. In: Geschichte und Gesellschaft 15, S. 563-79.

Bock, Gisela (1992): Ein Historikerinnenstreit? In: Geschichte und Gesellschaft 18, S. 400-04.

Bock, Gisela (1993): Gleichheit und Differenz in der nationalsozialistischen Rassenpolitik. In: Geschichte und Gesellschaft 19, S. 277-310.

Böltken, Andrea (1995): Führerinnen im Führerstaat. Pfaffenweiler: Centaurus.

Chatterjee, Partha (1993): The Nation and Its Fragments: Colonial and Postcolonial Histories. Princeton, New Jersey: Princeton New Jersey Press.

Chiteki Kyōryoku Kaigi (Hg.) (1943): Kindai no chōkoku [Überwindung der Moderne]. Tokio: Sōgensha.

Connell, Robert W. (2000): Der gemachte Mann: Konstruktion und Krise von Männlichkeiten (2. Aufl.). Opladen: Leske und Budrich.

Conrad, Sebastian (2002): Doppelte Marginalisierung: Plädoyer für eine transnationale Perspektive auf die deutsche Geschichte. In: Geschichte und Gesellschaft 28, S. 145-69.

de Bary, Brett (1997): Guest Editor's Introduction. In: U.S.-Japan Women's Journal. English Supplement 12, S. 3-16.

Doak, Kevin (1996): Ethnic nationalism and romanticism in early twentieth-century Japan. In: Journal of Japanese Studies 22 (1), S. 77-103.

Doak, Kevin (1997): What is a nation and who belongs? National narratives and the ethnic imagination in twentieth-century Japan. In: American Historical Review 102 (2), S. 283-309.

Enloe, Cynthia (1989): Bananas, Beaches, and Bases: Making Feminist Sense of International Politics. Berkeley: University of California Press.

Fujin Sensen [Frauenfront] (1984) [1930-31] 16 Hefte. Faksimile. Tokio: Ryokuin Shobō.

Fujitani, Takashi (1993): Inventing, forgetting, remembering: Toward a historical ethnography of the nation-state. In: Harumi Befu (Hg.): Cultural Nationalism in East Asia: Representation and Identity. Berkeley: University of California Press, S. 77-106.

Furukubo, Sakura (1991): Raichō no ,bosei-shugi' o yomu: Bosei o kijiku ni shita feminizumu saikō no tame ni [Raichōs Maternalismus wiedergelesen: Für eine erneute Betrachtung des maternalistischen Feminismus]. In: Josei-gaku Nenpō 12, S. 75-83.

Garon, Sheldon (1993): Women's groups and the Japanese state: Contending approaches to political integration, 1890-1945. In: Journal of Japanese Studies 19 (1), S. 5-41.

Gauntlett, John Owen und Robert K. Hall (Hg.) (1949): Kokutai no hongi: Cardinal Principles of the National Entity of Japan. Übers. v. John Owen und Robert K. Hall. Cambridge, Mass.: Harvard University Press. 
Gellner, Ernest (1983): Nations and Nationalism. Ithaka, NY: Cornell University Press.

Germer, Andrea (1996): „Genesis der Liebe“: Die sozialphilosophischen und feministischen Anschauungen Takamure Itsues. In: Bochumer Jahrbuch zur Ostasienforschung 20, S. 119-86.

Germer, Andrea (2000): Continuity and Change in Japanese Feminist Magazines: Fujin Sensen (1930-31) and Onna Erosu (1973-82). In: U. Wöhr, B. Hamill Sato und S. Suzuki (Hg.): Gender and Modernity: Rereading Japanese Women's Magazines (International Symposium in Europe, October 12-15, Belgium). Kioto: International Research Center for Japanese Studies, S. 101-30.

Germer, Andrea (2003): Historische Frauenforschung in Japan: Die Rekonstruktion der Vergangenheit in Takamure Itsues "Geschichte der Frau“ (Josei no rekishi). München: Iudicium.

Getreuer-Kargl, Ingrid (1997): Geschlechterverhältnis und Modernisierung. In: I. Lenz und M. Mae (Hg.): Getrennte Welten, gemeinsame Moderne? Geschlechterverhältnisse in Japan. Opladen: Leske und Budrich, S. 19-58.

Hane, Mikiso (1988): Reflections on the Way to the Gallows: Rebel Women in Prewar Japan. Berkeley et al.: University of California Press.

Harootunian, Harry D. (1979): Commentary on nationalism in Japan: Nationalism as intellectual history. In: Journal of Asian Studies XXXIX (1), S. 57-62.

Hausen, Karin (1992): Öffentlichkeit und Privatheit: Gesellschaftspolitische Konstruktionen und die Geschichte der Geschlechterbeziehungen. In: K. Hausen und H. Wunder (Hg.): Frauengeschichte - Geschlechtergeschichte. Frankfurt a. M. und New York: Campus, S. 81-88.

Havens, Thomas R. H. (1975): Women and war in Japan, 1937-45. In: The American Historical Review 80 (4), S. 913-34.

Hiratsuka, Raichō (1988a [1918]): Bosei hogo mondai ni tsuite futatabi Yosano Akiko-shi ni yosu [Nochmals an Yosano Akiko über das Problem des Mutterschutzes]. In: Nobuko Kōuchi (Hg.): Shiryō: Bosei hogo ronsō [Materialien: Die Mutterschutzdebatte]. Tokio: Domesu Shuppan, S. 105-16 (Original in Fujin Kōron, 3 (7)).

Hiratsuka, Raichō (1988b [1918]): Bosei hogo no shuchō wa iraishugi ka. Yosano, Kaetsu nishi e [Bedeutet der Ruf nach Mutterschutz den Ruf nach Abhängigkeit? An Frau Yosano und Frau Kaetsu]. In: Nobuko Kōuchi (Hg.): Shiryō: Bosei hogo ronsō [Materialien: Die Mutterschutzdebatte]. Tokio: Domesu Shuppan, S. 86-91 (Original in Fujin Kōron 3 (5)).

Igeta, Midori (Hg.) (2000): „Nihon“ kokka to onna [Der japanische Staat und die Frauen]. Tokio: Seikyūsha.

Kandiyoti, Deniz (1989): Women and the Turkish state: Political actors or symbolic pawns? In: N. Yuval-Davis und F. Anthias (Hg.): Woman - Na- 
tion - State. Basingstoke et al.: Macmillan, S. 126-49.

Kandiyoti, Deniz (1991): End of empire: Islam, nationalism, and women in Turkey. In: D. Kandiyoti (Hg.): Women, Islam, and the State. Philadelphia: Temple University Press, S. 22-47.

Kano, Masanao (1983): Kindai Nihon no minkangaku [Laienforschung im modernen Japan]. Tokio: Iwanami Shoten.

Kano, Masanao und Kiyoko Horiba (1985): Takamure Itsue [Takamure Itsue]. Tokio: Asahi Shinbunsha.

Kanō, Mikiyo (1989): ,Ōmikokoro' to ,hahagokoro': ,Yasukuni no haha' o umidashita mono [Das ,kaiserliche Herz' und das ,mütterliche Herz': Was die ,Mütter des Yasukuni-Schreines' hervorgebracht haben]. In: Mikiyo Kanō (Hg.): Josei to tennōsei [Die Frauen und das Tenno-System]. Tokio: Shisō no Kagakusha, S. 64-81.

Kanō, Mikiyo (2000): Okuni no tame ni shinu koto to umu koto [Für das Land sterben und für das Land gebären]. In: Midori Igeta (Hg.): „Nihon“ kokka to onna [Der japanische Staat und die Frauen]. Tokio: Seikyūsha, S. 89-124.

Karlin, Jason (2002): The gender of nationalism: Competing masculinities in Meiji Japan. In: Journal of Japanese Studies 28 (1), S. 41-78.

Katzoff, Beth (2000): For the Sake of the Nation, for the Sake of Women: The Pragmatism of Japanese Feminisms in the Asia-Pacific War (1931-1945). Ann Arbor: UMI.

Kawai, Yōko (1990): Dokusen Nihon no keisei to jokō no kekkaku [Formierung des Monopolkapitals und Tuberkulose unter den Arbeiterinnen]. In: Joseishi Sōgō Kenkyūkai (Hg.): Nihon joseishi [Frauengeschichte in Japan], Bd. 5. Tokio: Tōkyō Daigaku Shuppankai, S. 39-73.

Kawanishi, Jitsuzō (1943): Kessenka ni okeru Dai Nippon Fujinkai no shimei (shō) [Die Bestimmung der Großjapanischen Frauenvereinigung im Entscheidungskrieg (gekürzte Fassung)]. In: Yūko Suzuki (Hg.) (1995): Nihon josei undō shiryō shūsei. [Materialien zur Japanischen Frauenbewegung], Bd. 10. Tokio: Fuji Shuppan, S. 432-54.

Kessler, Christine (1993): Yamakawa Kikue und ihre Rolle in der Frauenbewegung der frühen Taishō-Zeit. Unveröffentlichte Magistra-Arbeit. Heidelberg: Japanologisches Seminar.

Key, Ellen (1905): Über Liebe und Ehe: Essays von Ellen Key (10. Auflage). Berlin: Fischer.

Kojima, Masaru (1993): „Dai Tōa kyōeiken“ e no shisō: Nihon seishin to Ajia-shugi o chūshin toshite [Gedankengut der "Großostasiatischen Wohlstandssphäre": Die japanische Seele und der Asianismus]. In: Y. Yamaguchi und R. Ruprecht (Hg.): Rekishi to aidentiti: Nihon to Doitsu ni totte no senkyūkyaku yonjūgonen [Geschichte und Identität: Das Jahr 1945 in Japan und Deutschland]. Tokio: Shibunkaku Shuppan, S. 307-17. 
Koonz, Claudia (1987): Mothers in the Fatherland. New York: St. Martin's Press.

Koonz, Claudia (1992): Erwiderung auf Gisela Bocks Rezension von Mothers in the Fatherland. In: Geschichte und Gesellschaft 18, S. 394-99.

Kōuchi, Nobuko (Hg.) (1988): Shiryō: Bosei hogo ronsō [Materialien: Die Mutterschutzdebatte]. Tokio: Domesu Shuppan.

Koyama, Shizuko (1982): Takamure Itsue ni okeru josei kaihō shisō no keisei to hatten [Form und Entwicklung von Takamure Itsues Denken über die Frauenbefreiung]. In: Shakai Shisōshi Kenkyū 6, S. 99-115.

Koyama, Shizuko (1991): Ryōsai kenbo to iu kihan [Die Norm der guten Ehefrau und weisen Mutter]. Tokio: Keisō Shobō.

Mamozai, Martha (1990): Komplizinnen. Hamburg: Rowohlt.

Matsumoto, Shigeru (1970): Motoori Norinaga. Cambridge, MA: Harvard University Press.

May, Katharina (1975): Die Erneuerung der Tanka-Poesie in der Meiji-Zeit (1868-1912) und die Lyrik Yosano Akikos. Wiesbaden: Harrassowitz.

McClintock, Anne (1997): „No longer in a future heaven“: Gender, race, and nationalism. In: A. McClintock, A. Mufti und E. Shohat (Hg.): Dangerous Liaisons: Gender, Nation, and Postcolonial Perspectives. Minneapolis: University of Minnesota Press, S. 89-112.

Mies, Maria (1990): Patriarchat und Kapital: Frauen in der internationalen Arbeitsteilung. Zürich: Rotpunkt.

Mitsui, Reiko (Hg.) (1963): Gendai fujin undōshi nenpyō [Tabellarische Chronologie der Frauenbewegung der Gegenwart]. Tokio: San'ichi Shobō.

Miyake, Yoshiko (1991): Doubling expectations: Motherhood and women's factory work under state management in Japan in the 1930s and 1940s. In: Gail Lee Bernstein (Hg.): Recreating Japanese Women, 16001945. Berkeley et al.: University of California Press, S. 267-95.

Mosse, George L. (1997): Das Bild des Mannes: Zur Konstruktion der modernen Männlichkeit. Frankfurt a. M.: Fischer.

Nagahara, Kazuko (1985): Josei tōgō to bosei: Kokka ga kitai suru boseizō [Die Integration von Frauen und Mutterschaft: Die vom Staat erwartete Vorstellung vom Mütterlichen]. In: H. Wakita (Hg.): Bosei o tou [Die Infragestellung des Mütterlichen], Bd. 2. Kioto: Jinbun Shoin, S. 192218.

Nagahara, Kazuko (1988): Takamure Itsue kenkyū ni manabu mono: Senzen senjiki no Takamure Itsue ni tsuite [Was wir aus der TakamureItsue-Forschung lernen können: Über Takamure Itsue in der Vorkriegsund Kriegszeit]. In: Rekishi Hyōron 455 (3), S. 40-58.

Neuss, Margret (1971): Die Seitōsha: Der Ausgangspunkt der japanischen Frauenbewegung in seinen zeitgeschichtlichen und sozialen Bedingungen. In: Oriens extremus XVIII, S. 1-66 (Teil 1), S. 137-201 (Teil 2). 
Nishikawa, Yūko (1975): Takamure Itsue to „Fujin Sensen“ [Takamure Itsue und die „Frauenfront"]. In: Shisō 609 (März), S. 368-89.

Nishikawa, Yūko (1990 [1982]): Sensō e no keika to yokusan no fujin [Die Entwicklung zum Krieg und die Frauen der Unterstützungskomitees]. In: Joseishi Sōgō Kenkyūkai (Hg.): Nihon joseishi [Frauengeschichte in Japan], Bd. 5. Tokio: Tōkyō Daigaku Shuppankai, S. 227-64.

Nishikawa, Yūko (1997): Japan's entry into the war and the support of women. In: US-Japan Women's Journal 12, S. 48-83.

Oguma, Eiji (2002): A Genealogy of Japanese Self-images. Melbourne: Trans Pacific Press.

Ōkawa, Shūmei (1939): Nihon 2600 nenshi [2600jährige Geschichte Japans]. Tokio: Dai Ichi Shobō.

Radhakrishnan, R. (1992): Nationalism, Gender, and the Narrative of Identity. In: Andrew Parker et al. (Hg.): Nationalisms and Sexualities. London and New York: Routledge, S. 77-95.

Schaarschmidt, Siegfried (Hg.) (1984): Schrei nach Frieden: Japanische Zeugnisse gegen den Krieg. Übers. v. Siegfried Schaarschmidt. Wien: Econ.

Schneider, Michael (2007): Were women Pan-Asianists the worst? Internationalism and Pan-Asianism in the careers of Inoue Hideko and Inoue Masaji. In: S. Saaler und J.V. Koschman (Hg.): Pan-Asianism in Modern Japanese History: Colonialism, Regionalism and Borders. New York: Routledge, S. 115-29.

Sievers, Sharon L. (1983): Flowers in Salt: The Beginnings of Feminist Consciousness in Modern Japan. Stanford, Cal.: Stanford University Press.

Smith, Anthony (2001): Nationalism: Theory, Ideology, History. Cambridge: Polity.

Suzuki, Yūko (1992): Jūgun ianfu, naisen kekkon: Sei no shinryaku, sengo sekinin o kangaeru [Trostfrauen und koreanisch-japanische Ehen: Nachdenken über sexuelle Invasion und Kriegsverantwortung]. Tokio: Miraisha.

Suzuki, Yūko (1995a): Bosei sensō heiwa: ,Nihonteki bosei' to feminizumu [Mütterlichkeit, Krieg, Frieden: ,Japanische Mütterlichkeit' und Feminismus]. In: Mikiyo Kanō (Hg.): Bosei fashizumu [Mütterlichkeitsfaschismus] (= Nyū feminizumu rebyū, Bd. 6). Tokio: Gakuyō Shobō, S. 68-73.

Suzuki, Yūko (Hg.) (1995b): Nihon josei undō shiryō shūsei [Materialien zur Japanischen Frauenbewegung], Bd. 10. Tokio: Fuji Shuppan.

Takamure, Itsue (1926a): Ren'ai sōsei [Genesis der Liebe]. In: Kenzō Hashimoto (Hg.): Takamure Itsue zenshū [Takamure Itsue Gesamtausgabe], Bd. 7. Tokio: Rironsha, S. 7-213.

Takamure, Itsue (1926b): Shin joseishugi no teishō [Vorschlag einer neuen Weiblichkeitsidee]. In: Kaihō 6, S. 3-33.

Takamure, Itsue (1934): Nihon seishin ni tsuite [Über die japanische Seele]. In: Fujo Shinbun (12. August). Abgedr. in: N. Kōno et al. (Hg.) (1979): 
Takamure Itsue ronsh $\bar{u}$ [Studien zu Takamure Itsue]. Tokio: JCA Shuppan, S. 205-08.

Takamure, Itsue (1937): Tenki ni tatsu fujin [Die Frau am Wendepunkt]. In: Fujo Shinbun (26. Dezember). Abgedr. in: N. Kōno et al. (Hg.) (1979): Takamure Itsue ronsh $\bar{u}$ [Studien zu Takamure Itsue]. Tokio: JCA Shuppan, S. 209-10.

Takamure, Itsue (1940): Josei 2600 nenshi [2600jährige Geschichte der Frau]. Tokio: Kōseikaku.

Takamure, Itsue (1943): Gunji to josei [Das Militär und die Frauen]. In: Nippon Fujin 2 (1) (November), S. 8-11.

Takamure, Itsue (1944a): Kamigokoro [Das göttliche Herz]. In: Nippon Fujin 2 (8) (September), S. 8-11.

Takamure, Itsue (1944b): Kagezen [Mahl für die Abwesenden]. In: Nippon Fujin 2 (6) (Juli), S. 8-11.

Takamure, Itsue (1944c): Taoyame [Sanfte Weiblichkeit]. In: Nippon Fujin 2 (10) (November), S. 8-11.

Takamure, Itsue (1954-58): Josei no rekishi [Geschichte der Frau]. Tokio: Kōdansha.

Takeuchi, Yoshimi (1987): Kindai no chōkoku [Überwindung der Moderne]. In: T. Kawakami und Y. Takeuchi (Hg.): Kindai no chōkoku [Überwindung der Moderne]. Tokio: Fuzanbō, S. 273-341.

Thomas, Julia Adeney (1998): Naturalizing nationhood: Ideology and practice in early twentieth-century Japan. In: S.A. Minichiello (Hg.): Japan's Competing Modernities: Issues in Culture and Democracy 1900-1930. Honolulu: University of Hawai'i Press, S. 114-32.

Toby, Ronald P. (2001): Rescuing the nation from history: The state of the state in early modern Japan. In: Monumenta Nipponica 56 (2), S. 197-237.

Tomida, Hiroko (2004): Hiratsuka Raichō and Early Japanese Feminism. Leiden, Boston: Brill.

Tsunoda, Ryusaku et al. (1958): Sources of Japanese Tradition. New York, London: Columbia University Press.

Tsurumi, Patricia (1985): Feminism and anarchism in Japan: Takamure Itsue, 1894-1964. In: Bulletin of Concerned Asian Scholars 17 (2), S. 2-19.

Ueno, Chizuko (2004): Nationalism and Gender. Melbourne: Trans Pacific Press.

Wilson, Sandra (2006): Family or state? Nation, war, and gender in Japan, 1937-45. In: Critical Asian Studies 38 (2), S. 209-38.

Yamada, Waka (1988 [1918]): Kongo no fujin mondai o teishō su [Vorschläge für die künftige Frauenfrage]. In: Nobuko Kōuchi (Hg.): Shiryō: Bosei hogo ronsō [Materialien: Die Mutterschutzdebatte]. Tokio: Domesu Shuppan, S. 91-95.

Yamakawa, Kikue (1988a [1918]): Bosei hogo to keizaiteki dokuritsu. Yo- 
sano, Hiratsuka nishi no ronsō [Mutterschutz und wirtschaftliche Selbständigkeit: Die Debatte zwischen Frau Yosano und Frau Hiratsuka]. In: Nobuko Kōuchi (Hg.): Shiryō: Bosei hogo ronsō [Materialien: Die Mutterschutzdebatte]. Tokio: Domesu Shuppan, S. 132-46 (Original in Fujin Kōron 3 (9) September).

Yamakawa, Kikue (1988b [1918]): Fujin o uragiru fujinron o hyōsu [Eine Kritik am Verrat an den Frauen in manchen Frauenbefreiungstheorien]. In: Nobuko Kōuchi (Hg.): Shiryō: Bosei hogo ronsō [Materialien: Die Mutterschutzdebatte]. Tokio: Domesu Shuppan, S. 117-31 (Original in Shin Nihon 8 (8) August).

Yamashita, Etsuko (1988): Takamure Itsue ron: ,Haha' no arukeoroj̄̄ [Abhandlung über Takamure Itsue: Eine Archäologie der „Mutter“]. Tokio: Kawade Shobō Shinsha.

Yoneda, Sayoko (1990 [1982]): Boseishugi no rekishiteki igi [Die historische Bedeutung des Maternalismus]. In: Joseishi Sōgō Kenkyūkai (Hg.): Nihon joseishi [Frauengeschichte in Japan], Bd. 5. Tokio: Tōkyō Daigaku Shuppankai, S. 115-48.

Yosano, Akiko (1988a) [1918]: Joshi no shokugyōteki dokuritsu o gensoku to seyo [Für den Grundsatz der beruflichen Unabhängigkeit der Frauen]. In: Nobuko Kōuchi (Hg.): Shiryō: Bosei hogo ronsō [Materialien: Die Mutterschutzdebatte]. Tokio: Domesu Shuppan, S. 81-84 (Original in Jogaku Sekai 18 (1) Januar).

Yosano, Akiko (1988b) [1918]: Joshi no tettei shita dokuritsu [Die vollkommene Unabhängigkeit der Frau]. In: Nobuko Kōuchi (Hg.): Shiryō: Bosei hogo ronsō [Materialien: Die Mutterschutzdebatte]. Tokio: Domesu Shuppan, S. 85-86 (Original in Fujin Kōron 3 (3) März).

Yosano, Akiko (1988c [1918]): Hiratsuka, Yamakawa, Yamada san joshi ni kotau [Antworten auf Frau Hiratsuka, Frau Yamakawa und Frau Yamada]. In: Nobuko Kōuchi (Hg.): Shiryō: Bosei hogo ronsō [Materialien: Die Mutterschutzdebatte]. Tokio: Domesu Shuppan, S. 176-92 (Original in Taiyō 24 (13) Februar).

Yuval-Davis, Nira (1997): Gender \& Nation. London et al.: SAGE Publications.

Yuval-Davis, Nira und Floya Anthias (Hg.) (1989): Woman - Nation - State. Basingstoke et al.: Macmillan. 